\title{
On operators from separable reflexive spaces with asymptotic structure
}

\author{
by \\ Bentuo Zheng (Austin, TX)
}

\begin{abstract}
Let $1<q<p<\infty$ and $q \leq r \leq p$. Let $X$ be a reflexive Banach space satisfying a lower- $\ell_{q}$-tree estimate and let $T$ be a bounded linear operator from $X$ which satisfies an upper- $\ell_{p}$-tree estimate. Then $T$ factors through a subspace of $\left(\sum F_{n}\right)_{\ell_{r}}$, where $\left(F_{n}\right)$ is a sequence of finite-dimensional spaces. In particular, $T$ factors through a subspace of a reflexive space with an $\left(\ell_{p}, \ell_{q}\right)$ FDD. Similarly, let $1<q<r<p<\infty$ and let $X$ be a separable reflexive Banach space satisfying an asymptotic lower- $\ell_{q}$-tree estimate. Let $T$ be a bounded linear operator from $X$ which satisfies an asymptotic upper- $\ell_{p}$-tree estimate. Then $T$ factors through a subspace of $\left(\sum G_{n}\right)_{\ell_{r}}$, where $\left(G_{n}\right)$ is a sequence of finite-dimensional spaces. In particular, $T$ factors through a subspace of a reflexive space with an asymptotic $\left(\ell_{p}, \ell_{q}\right)$ FDD.
\end{abstract}

1. Introduction. In [10], E. Odell and Th. Schlumprecht prove that a separable reflexive Banach space $X$ which satisfies an $\left(\ell_{p}, \ell_{q}\right)$-tree estimate for $1 \leq q \leq p \leq \infty$ embeds into a reflexive Banach space with an $\left(\ell_{p}, \ell_{q}\right)$ FDD. In particular, this proves that if every normalized weakly null tree in a separable reflexive Banach space $X$ has a branch equivalent to the unit vector basis of $\ell_{p}(1<p<\infty)$, then $X$ is isomorphic to a subspace of an $\ell_{p}$ sum of finite-dimensional spaces. Then, in [11], E. Odell, Th. Schlumprecht and A. Zsák prove that a separable reflexive Banach space $X$ which satisfies an asymptotic $\left(\ell_{p}, \ell_{q}\right)$-tree estimate embeds into a reflexive Banach space with an asymptotic $\left(\ell_{p}, \ell_{q}\right)$ FDD. So a special case is that every separable reflexive asymptotic $\ell_{p}$ space is a subspace of a reflexive Banach space with an asymptotic $\ell_{p}$ FDD.

Recall [8] that a Banach space $X$ with an FDD $\left(E_{n}\right)$ is asymptotic $\ell_{p}$ with respect to $\left(E_{n}\right)$ if there exists a $1 \leq C<\infty$ so that for all $n$, every

2000 Mathematics Subject Classification: Primary 46B03; Secondary 46B20.

Key words and phrases: lower- $\ell_{p}$-tree estimate, upper- $\ell_{q}$-tree estimate, asymptotic lower- $\ell_{p}$-tree estimate, asymptotic upper- $\ell_{q}$-tree estimate, factorizations.

Supported in part by NSF/DMS 02-00690 and 05-03688. This paper forms part of the author's doctoral dissertation which is being prepared at Texas A\&M University under the direction of W. B. Johnson. 
normalized block sequence $\left(x_{i}\right)_{i=1}^{n}$ of $\left(E_{i}\right)_{i=n}^{\infty}$ is $C$-equivalent to the unit vector basis of $\ell_{p}$. A coordinate-free version of this notion is in [7]. Let $X$ be an arbitrary Banach space. Then $X$ is said to be asymptotic $\ell_{p}$ if there exists $1 \leq C<\infty$ so that $\forall n \in \mathbb{N}, \exists Y_{1} \in \operatorname{cof}(X) \forall y_{1} \in S_{Y_{1}}, \ldots, \exists Y_{n} \in \operatorname{cof}(X)$ $\forall y_{n} \in S_{Y_{n}},\left(y_{i}\right)_{i=1}^{n}$ is $C$-equivalent to the unit vector basis of $\ell_{p}^{n}$. An FDD $\left(E_{n}\right)_{i=1}^{\infty}$ is asymptotic $\left(\ell_{p}, \ell_{q}\right)$ if there exists $0<C<\infty$ such that for all $n \in \mathbb{N}$ and all block sequences $\left(x_{i}\right)_{i=1}^{n}$ of $\left(E_{n}\right)_{i=n}^{\infty}$,

$$
C^{-1}\left(\sum_{i=1}^{n}\left\|x_{i}\right\|^{p}\right)^{1 / p} \leq\left\|\sum_{i=1}^{n} x_{i}\right\| \leq C\left(\sum_{i=1}^{n}\left\|x_{i}\right\|^{q}\right)^{1 / q} .
$$

If $p=q$, then we say that $\left(E_{n}\right)$ is asymptotic $\ell_{p}$.

The results above can be restated in the way that under some conditions, the identity operator on a separable reflexive Banach space factors through a subspace of an $\ell_{p}$ sum of finite-dimensional spaces, and under some other conditions, it factors through a subspace of a space with an asymptotic $\ell_{p}$ FDD. From this point of view, it is natural to consider general operators from a separable reflexive Banach space. The goal is to find the right conditions under which the operators factor through a subspace of an $\ell_{p}$ sum of finite-dimensional spaces or factor through a subspace of a space with an asymptotic $\ell_{p}$ FDD. In [12], the author proves that if $X$ is a Banach space with an FDD satisfying a block lower- $p$ estimate and $T$ is an operator from $X$ which satisfies an upper- $\ell_{p}$-tree estimate, then $T$ factors through $\left(F_{n}\right)_{\ell_{p}}$, where $\left(F_{n}\right)$ is a sequence of finite-dimensional spaces. An important consequence is that any bounded linear operator from $L_{p}(2<p<\infty)$ which satisfies an upper- $\ell_{p}$-tree estimate factors through $\ell_{p}$ (actually this is also a necessary condition).

In this paper, the author proves the following theorems. Theorem A generalizes the results in [12] we mentioned above and Theorem B gives conditions for operators from spaces with certain asymptotic structure to factor through a subspace of an $\ell_{r}$ sum of finite-dimensional spaces.

Theorem A. Let $1<q \leq r \leq p<\infty$ and let $X$ be a separable reflexive Banach space which satisfies a lower- $\ell_{q}$-tree estimate. Let $T$ be a bounded linear operator from $X$ into $Y$ which satisfies an upper- $\ell_{p}$-tree estimate. Then $T$ factors through a subspace of $\left(\sum F_{n}\right)_{\ell_{r}}$, where $\left(F_{n}\right)$ is a sequence of finite-dimensional spaces.

Theorem B. Let $1<q<r<p<\infty$ and let $X$ be a separable reflexive Banach space which satisfies an asymptotic lower- $\ell_{q}$-tree estimate. Let $T$ be a bounded linear operator from $X$ which satisfies an asymptotic upper- $\ell_{p^{-}}$ tree estimate. Then $T$ factors through a subspace of $\left(F_{n}\right)_{\ell_{r}}$, where $\left(F_{n}\right)$ is a sequence of finite-dimensional spaces. 
2. Definitions and notations. Let $X$ be a Banach space and let $S_{X}$ be the unit sphere of $X$. Let $[\mathbb{N}]^{<\omega}$ denote all finite subsets of the positive integers. By a normalized weakly null tree we mean a family $\left(x_{A}\right)_{A \in[\mathbb{N}]<\omega} \subset$ $S_{X}$ with the property that every sequence $\left(x_{A \cup\{n\}}\right)_{n \in \mathbb{N}}$ is weakly null. Let $A=\left\{n_{1}, \ldots, n_{m}\right\}$ with $n_{1}<\cdots<n_{m}$ and $B=\left\{j_{1}, \ldots, j_{r}\right\}$ with $j_{1}<\cdots<$ $j_{r}$. Then we say $A$ is an initial segment of $B$ if $m \leq r$ and $n_{i}=j_{i}$ when $1 \leq i \leq m$. The tree order on $\left(x_{A}\right)_{A \in[\mathbb{N}]<\omega}$ is given by $x_{A} \leq x_{B}$ if $A$ is an initial segment of $B$. A branch of a tree is a maximal linearly ordered subset of the tree under the tree order.

Definition 2.1. Let $V$ be a Banach space with a 1-unconditional and normalized basis $\left(v_{i}\right)$. We say that a Banach space $X$ satisfies a lower- $V$-tree estimate if there exists a $C \geq 1$ such that every normalized weakly null tree in $X$ has a branch $\left(x_{i}\right)$ so that for all $\left(a_{i}\right) \subset \mathbb{R}$,

$$
\left\|\sum a_{i} x_{i}\right\| \geq C^{-1}\left\|\sum a_{i} v_{i}\right\|
$$

In this paper, we need an analogous definition for operators.

Definition 2.2. Let $U$ be a Banach space with a 1-unconditional and normalized basis $\left(u_{i}\right)$. Let $T$ be a bounded linear operator from $X$. We say that $T$ satisfies an upper- $U$-tree estimate if there exists a $C>0$ such that every normalized weakly null tree in $X$ has a branch $\left(x_{i}\right)$ so that for all $\left(a_{i}\right) \subset \mathbb{R}$

$$
\left\|T\left(\sum a_{i} x_{i}\right)\right\| \leq C\left\|\sum a_{i} u_{i}\right\| .
$$

Definition 2.3. Let $1 \leq p \leq \infty$. A reflexive Banach space $X$ satisfies an asymptotic lower- $\ell_{p}$-tree estimate if there exists a $1 \leq C<\infty$ so that for every $k \in \mathbb{N}$, every normalized weakly null tree of length $k$ in $X$ has a branch $\left(x_{i}\right)_{i=1}^{k}$ such that for all $\left(a_{i}\right) \subset \mathbb{R}$,

$$
\left\|\sum_{i=1}^{k} a_{i} x_{i}\right\| \geq C^{-1}\left(\sum_{i=1}^{k}\left\|a_{i}\right\|^{p}\right)^{1 / p} .
$$

Definition 2.4. Let $1 \leq q \leq \infty$ and let $T$ be a bounded linear operator from a Banach space $X$. Then $T$ satisfies an asymptotic upper- $\ell_{q}$-tree estimate if there exists a $1 \leq C<\infty$ so that for every $k \in \mathbb{N}$, every normalized weakly null tree of length $k$ in $X$ has a branch $\left(x_{i}\right)_{i=1}^{k}$ such that

$$
\left\|\sum_{i=1}^{k} x_{i}\right\| \leq C\left(\sum_{i=1}^{k}\left\|x_{i}\right\|^{q}\right)^{1 / q} .
$$

3. Main results. Let $\left(u_{i}\right)$ be a sequence in a Banach space $U$ and let $\left(v_{i}\right)$ be a sequence in a Banach space $V$. We say that $\left(u_{i}\right) C$-dominates $\left(v_{i}\right)$ 
or $\left(v_{i}\right)$ is $C^{-1}$-dominated by $\left(u_{i}\right)$ if for all $\left(a_{i}\right) \subset \mathbb{R}$,

$$
\left\|\sum a_{i} u_{i}\right\|_{U} \geq C\left\|\sum a_{i} v_{i}\right\|_{V}
$$

Let $\left(w_{i}\right)$ be a 1-unconditional normalized basis for $W$. Let $F=\left(F_{n}\right)$ be an FDD for a Banach space $Z$. Then we define the space $Z_{W}(F)$ to be the completion of $c_{00}\left(\bigoplus F_{n}\right)$ under the norm

$$
\left\|\left(x_{i}\right)\right\|=\left\|\sum_{j}\right\| x_{i}\left\|w_{j}\right\|
$$

Let $\left(w_{i}\right)$ be a basis for some Banach space $W$. We say that $\left(w_{i}\right)$ is $C$ subsymmetric if it is 1-unconditional and every subsequence is $C$-equivalent to $\left(w_{i}\right)$.

Theorem 3.1. Let $U$ and $V$ be two Banach spaces with 1-unconditional normalized bases $\left(u_{i}\right)$ and $\left(v_{i}\right)$. Let $\left(w_{i}\right)$ be a normalized $C^{\prime}$-subsymmetric basis for $W$. Suppose that $\left(u_{i}\right) A_{1}$-dominates $\left(w_{i}\right)$ and $\left(w_{i}\right) A_{2}$-dominates $\left(v_{i}\right)$. Let $X$ be a separable reflexive Banach space with an FDD $\left(E_{n}\right)$ which satisfies a lower-U-tree estimate. Let $T$ be a bounded linear operator from $X$ into $Y$ which satisfies an upper- $V$-tree estimate. Then $T$ factors through $X_{W}(F)$, where $F=\left(F_{n}\right)$ is a blocking of $\left(E_{n}\right)$.

In the proof of Theorem 3.1, the following lemmas are used.

Lemma 3.2 (Propositions 2.4 and 2.5 in [10]). Let $X$ be a separable reflexive Banach space which embeds into a reflexive Banach space with an $F D D\left(E_{n}\right)$. Then for $\mathcal{A} \subset S_{X}^{\omega}$, the following are equivalent:

(a) For all $\varepsilon>0$, every weakly null tree in $S_{X}$ has a branch in $\widetilde{\mathcal{A}}_{\varepsilon}$.

(b) For all $\varepsilon>0$, there exists a blocking $\left(F_{n}\right)$ of $\left(E_{n}\right)$ and $\delta=\left(\delta_{i}\right)$ with $\delta_{i} \downarrow 0$ so that if $\left(x_{n}\right) \subset S_{X}$ is a $\delta$-skipped block sequence with respect to $\left(F_{i}\right)$ then $\left(x_{n}\right) \in \widetilde{\mathcal{A}}_{\varepsilon}$.

Here $\mathcal{A}_{\varepsilon}=\left\{\left(x_{n}\right) \subset S_{X}\right.$ : there exists $\left(y_{n}\right) \in \mathcal{A}$ with $\left\|x_{n}-y_{n}\right\|<\varepsilon / 2^{n}$ for all $n\}$ and $\widetilde{\mathcal{A}}_{\varepsilon}$ denotes the closure of $\mathcal{A}_{\varepsilon}$ with respect to the product topology of the discrete topology on $S_{X}$.

Given an FDD $\left(E_{n}\right),\left(x_{n}\right)$ is said to be a skipped block sequence with respect to $\left(E_{n}\right)$ if there exists a sequence of integers $1=m_{1}<m_{2}<\cdots$ such that $m_{n}+1<m_{n+1}$ and $x_{n} \in \bigoplus_{j=m_{n}+1}^{m_{n+1}-1} E_{j}, \forall n \in \mathbb{N}$. Let $\delta=\left(\delta_{i}\right)$ be a sequence of positive numbers decreasing to 0 . We say $\left(y_{n}\right)$ is a $\delta$-skipped block sequence with respect to $\left(E_{n}\right)$ if there is a skipped block sequence $\left(x_{n}\right)$ so that $\left\|y_{n}-x_{n}\right\|<\delta_{n}\left\|y_{n}\right\|$ for all $n \in \mathbb{N}$. We say $\left(F_{n}\right)$ is a blocking of $\left(E_{n}\right)$ if there is a sequence of integers $0=k_{0}<k_{1}<\cdots$ so that $F_{n}=\bigoplus_{j=k_{n-1}+1}^{k_{n}} E_{j}$.

Proof of Theorem 3.1. For a blocking $F=\left(F_{i}\right)$ of $E=\left(E_{n}\right)$, let $J_{F}$ be the natural embedding of $X$ into $X_{W}(F)$ so that if $x=\sum x_{i}$ with $x_{i} \in F_{i}$, 
then $J_{F}(x)=\sum x_{i} \in X_{W}(F)$. We define $\widetilde{T}$ to be the operator from $J_{F}(X)$ into $Y$ so that $\widetilde{T} \circ J_{F}=T$. Initially, $J_{F}$ and $\widetilde{T}$ are only defined on the linear span of the FDD's. Once they are bounded, they have bounded linear extensions to the closures. So our goal is to find an appropriate blocking $F$ of $E$ so that $J_{F}$ and $\widetilde{T}$ are bounded. Let $C \geq 1$ be a constant associated with the upper- $V$-tree estimate for the operator $T$ and set

$$
\mathcal{A}=\left\{\left(x_{i}\right) \in S_{X}^{\omega}: \forall j \in \mathbb{N},\left\|T\left(\sum_{i=1}^{j} a_{i} x_{i}\right)\right\| \leq C\left\|\sum_{i=1}^{j} a_{i} v_{i}\right\|, \forall\left(a_{i}\right) \subset \mathbb{R}\right\} .
$$

Since $T$ satisfies an upper- $V$-tree estimate, applying Lemma 3.2 to the set $\mathcal{A}$, we get a blocking $\left(G_{i}\right)$ of $\left(E_{i}\right)$ such that given $\varepsilon>0$, there exists $\delta=\left(\delta_{i}\right)$ so that if $\left(x_{n}\right) \subset S_{X}$ is a $\delta$-skipped block sequence with respect to $\left(G_{n}\right)$, then whenever $\sum a_{i} x_{i}$ converges, we have $\left\|T\left(\sum a_{i} x_{i}\right)\right\| \leq(1+\varepsilon) C\left\|\sum a_{i} v_{i}\right\|$.

Let $\widetilde{C}$ be a constant associated with the lower- $U$-tree estimate for $X$ and set

$$
\mathcal{B}=\left\{\left(x_{i}\right) \in S_{X}^{\omega}: \forall j \in \mathbb{N},\left\|\sum_{i=1}^{j} a_{i} x_{i}\right\| \geq \widetilde{C}^{-1}\left\|\sum_{i=1}^{j} a_{i} u_{i}\right\|, \forall\left(a_{i}\right) \subset \mathbb{R}\right\} .
$$

Since $X$ satisfies a lower- $U$-tree estimate, applying Lemma 3.2 again to the set $\mathcal{B}$ and properly shrinking $\delta$, we get a blocking $\left(H_{i}\right)$ of $\left(G_{i}\right)$ such that if $\left(x_{n}\right) \subset S_{X}$ is a $\delta$-skipped block sequence with respect to $\left(H_{n}\right)$, then whenever $\sum a_{i} x_{i}$ converges, we have $\left\|\sum a_{i} x_{i}\right\| \geq\left(\widetilde{C}^{-1} /(1+\varepsilon)\right)\left\|\sum a_{i} u_{i}\right\|$. From the above arguments, we get a blocking $\left(H_{i}\right)$ of $\left(E_{i}\right)$ so that $\left(u_{i}\right)$ is $\widetilde{C}^{-1} /(1+\varepsilon)$-dominated by any $\delta$-skipped block sequence with respect to $\left(H_{i}\right)$ in $X$ while the image of any $\delta$-skipped block sequence with respect to $\left(H_{i}\right)$ in $X$ under $T$ is $(1+\varepsilon) C$-dominated by $\left(v_{i}\right)$. Let $K=\sup _{m<n}\left\|P_{n}-P_{m}\right\|$ be the projection constant for $\left(E_{i}\right)$, where $P_{n}$ is the canonical projection from $X$ onto $\bigoplus_{i=1}^{n} E_{i}$. Using the "killing the overlap technique" [3], we can find a further blocking $F=\left(F_{n}\right)$ of $\left(H_{n}\right)$ with $F_{n}=\bigoplus_{j=l(n)+1}^{l(n+1)} H_{j}$, so that for any $x=\sum x_{j} \in S_{X}, x_{j} \in H_{j}$, there are $t_{n}$ 's with $l(n)<t_{n}<l(n+1)$ such that $\left\|x_{t_{j}}\right\|<\delta_{i}$, where $0=l(1)<l(2)<\cdots$.

First, we prove $\widetilde{T}$ is bounded. Let $\varepsilon>0$ and let $\sum \delta_{i}<\varepsilon$. Without loss of generality, we assume $\|T\|=1$. Let $x=\sum x_{i}=\sum \widetilde{x}_{j} \in S_{X}$ with $x_{i} \in F_{i}$ and $\widetilde{x}_{j} \in H_{j}$. Then

$$
\begin{aligned}
\|\widetilde{T}(x)\| & =\left\|T\left(\sum x_{i}\right)\right\| \leq\left\|T\left(\sum x_{2 i}\right)\right\|+\left\|T\left(\sum x_{2 i-1}\right)\right\| \\
& \leq(1+\varepsilon) C\left(\left\|\sum\right\| x_{2 i}\left\|v_{i}\right\|+\left\|\sum_{i>1}\right\| x_{2 i-1}\left\|v_{i}\right\|+\left\|x_{1}\right\|\right) \\
& \leq(1+\varepsilon) C A_{2}^{-1}\left(\left\|\sum\right\| x_{2 i}\left\|w_{i}\right\|+\left\|\sum_{i>1}\right\| x_{2 i-1}\left\|w_{i}\right\|+\left\|x_{1}\right\|\right)
\end{aligned}
$$




$$
\begin{aligned}
& \leq(1+\varepsilon) C C^{\prime} A_{2}^{-1}\left(\left\|\sum\right\| x_{2 i}\left\|w_{2 i}\right\|+\left\|\sum_{i>1}\right\| x_{2 i-1}\left\|w_{2 i-1}\right\|+\left\|x_{1}\right\|\right) \\
& \leq(1+\varepsilon) C C^{\prime} A_{2}^{-1}\left(\left\|\sum\right\| x_{i}\left\|w_{i}\right\|+\left\|\sum\right\| x_{i}\left\|w_{i}\right\|+\left\|\sum\right\| x_{i}\left\|w_{i}\right\|\right) \\
& =3(1+\varepsilon) C C^{\prime} A_{2}^{-1}\|x\|_{X_{W}(F)} .
\end{aligned}
$$

Hence $\widetilde{T}$ is bounded. It remains to prove that $J_{F}$ is bounded. Let $t_{0}=1$ and let $y_{i}=\sum_{j=t_{i-1}}^{t_{i}-1} \widetilde{x}_{j}$. Define $\widetilde{y}_{1}=y_{1}$ and $\widetilde{y}_{i}=y_{i}-\widetilde{x}_{t_{i-1}}$ for $i \geq 2$. Then

$$
\begin{aligned}
\|x\|_{X_{W}(F)} & =\left\|\sum_{i}\right\| x_{i}\left\|w_{i}\right\| \leq A_{1}^{-1}\left\|\sum_{i}\right\| x_{i}\left\|u_{i}\right\| \\
& \leq A_{1}^{-1}\left\|\sum_{i} K\left(\left\|y_{i}\right\|+\left\|y_{i+1}\right\|\right) u_{i}\right\| \\
& \leq K A_{1}^{-1}\left(\left\|\sum_{i>1}\right\| y_{i}\left\|u_{i}\right\|+\left\|\sum\right\| y_{i+1}\left\|u_{i}\right\|+\left\|y_{1}\right\|\right) \\
& \leq K A_{1}^{-1}\left(\left\|\sum_{i>1}\right\| \widetilde{y}_{i}\left\|u_{i}\right\|+\left\|\sum\right\| \widetilde{y}_{i+1}\left\|u_{i}\right\|+\left\|y_{1}\right\|+2 \varepsilon\right) \\
& \leq(1+\varepsilon) K A_{1}^{-1} \widetilde{C}\left(\left\|\sum_{i>1} \widetilde{y}_{i}\right\|+\left\|\sum \widetilde{y}_{i+1}\right\|+\widetilde{C}^{-1}\left\|y_{1}\right\|+2 \widetilde{C}^{-1} \varepsilon\right) \\
& \leq(1+\varepsilon) K A_{1}^{-1} \widetilde{C}\left(K(\|x\|+\varepsilon)+K(\|x\|+\varepsilon)+\widetilde{C}^{-1} K\|x\|+2 \widetilde{C}^{-1} \varepsilon\right) \\
& \leq(1+\varepsilon) K\left(2 K+2 K \varepsilon+\widetilde{C}^{-1} K+2 \widetilde{C}^{-1} \varepsilon\right) A_{1}^{-1} \widetilde{C}\|x\| .
\end{aligned}
$$

For the last step, we use the condition that $x \in S_{X}$. So $J_{F}$ is bounded.

Theorem 3.1 concerns operators from spaces with an FDD. For operators from spaces without an FDD, we have the following corollaries.

Corollary 3.3. Let $\left(u_{i}\right)$ be a normalized 1-subsymmetric basis for a reflexive space $U$. Let $\left(v_{i}\right)$ be a normalized 1-subsymmetric basis for a reflexive space $V$ and let $\left(w_{i}\right)$ be a normalized subsymmetric basis for a reflexive space $W$. Suppose that $\left(u_{i}\right)$ dominates $\left(w_{i}\right)$ and $\left(w_{i}\right)$ dominates $\left(v_{i}\right)$. Also assume that $\left(u_{i}\right)$ is dominated by every normalized block basis of $\left(u_{i}\right)$ and $\left(v_{i}^{*}\right)$ is dominated by every normalized block basis of $\left(v_{i}^{*}\right)$. Let $X$ be a separable reflexive Banach space which satisfies a lower-U-tree estimate and let $Y$ be a separable reflexive Banach space which satisfies an upper-V-tree estimate. Then any bounded linear operator $T$ from $X$ into $Y$ factors through a subspace of $Z_{W}(F)$, where $F=\left(F_{n}\right)$ is a sequence of finite-dimensional spaces.

Proof. By Theorem 4.4 in [11], $Y$ embeds into a reflexive space $\widetilde{Y}$ with an FDD $\left(G_{n}\right)$ which satisfies an upper- $V$-tree estimate. We use $\widetilde{U}, \widetilde{V}$ and $\widetilde{W}$ to denote the closed linear spans of $\left(u_{i}^{*}\right),\left(v_{i}^{*}\right)$ and $\left(w_{i}^{*}\right)$ respectively, where $\left(u_{i}^{*}\right),\left(v_{i}^{*}\right)$ and $\left(w_{i}^{*}\right)$ are the biorthogonal functionals of $\left(u_{i}\right),\left(v_{i}\right)$ and $\left(w_{i}\right)$ 
respectively. Let $T^{*}$ be the adjoint operator of $T$. Since the image of $T^{*}$ is inside $X^{*}$ which satisfies an upper- $\widetilde{U}$-tree estimate (Corollary 4.3 in [11]), $T^{*}$ satisfies an upper- $\widetilde{U}$-tree estimate. As $\widetilde{Y}$ satisfies an upper- $V$-tree estimate, $\widetilde{Y}^{*}$ satisfies a lower- $\widetilde{V}$-tree estimate. By Theorem $3.1, T^{*}$ factors through $Z_{\widetilde{W}}\left(\widetilde{F}_{n}\right)$. By considering $T^{* *}$, which is $T$, we conclude that $T$ factors through a subspace of $Z_{\widetilde{W}}\left(\widetilde{F}_{n}\right)^{*}$, which is $Z_{W}(F)$.

Proof of Theorem A. By Theorem 2.1 in [10], $X$ is a quotient of a reflexive space $Z$ with an FDD which satisfies a lower- $\ell_{q}$-tree estimate. Let $Q$ be a quotient map from $Z$ onto $X$. Then it is easy to see that $T \circ Q$ satisfies an upper- $\ell_{p}$-tree estimate. By Theorem 3.1, $\widetilde{T}=T \circ Q$ factors through $\left(E_{n}\right)_{\ell_{r}}$, where $\left(E_{n}\right)$ is a sequence of finite-dimensional spaces. Let $\widetilde{T}^{*}$ be the adjoint operator of $\widetilde{T}$. Then $\widetilde{T}^{*}=J \circ T^{*}$ factors through $\left(E_{n}^{*}\right)_{\ell_{r^{\prime}}}$, where $J$ is an embedding of $X^{*}$ into $Z^{*}$ and $1 / r^{\prime}+1 / r=1$. This implies that $T^{*}$ factors through a subspace $H$ of $\left(E_{n}^{*}\right)_{\ell_{r^{\prime}}}$. By [5], $H$ is also a quotient of some $\left(F_{n}\right)_{\ell_{r^{\prime}}}$. By considering $T^{* *}$, which is $T$, we deduce that $T$ factors through a subspace of $\left(F_{n}^{*}\right) \ell_{r}$.

Let $1<p<\infty$ and let $\ell_{p, 1}$ be the completion of $c_{00}$ under

$$
\left\|\left(a_{i}\right)\right\|_{\ell_{p, 1}}=\sum i^{1 / p-1} a_{i}^{*}
$$

where $\left(a_{i}^{*}\right)$ is the decreasing rearrangement of $\left(\left|a_{i}\right|\right)$.

REMARK 3.4. Let $1 \leq r<p \leq \infty$. Then the canonical basis of $\ell_{r}$ dominates the canonical basis of $\ell_{p, 1}$.

The Tsirelson space corresponding to $p$ and $0<\gamma<1$ is denoted by $T\left(\ell_{p}, \gamma\right)$ (see X.E in [1]). The standard Tsirelson space is $T=T\left(\ell_{1}, 1 / 2\right)$ and the original $p$-convexification of $T$ is $T\left(\ell_{p}, 2^{-1 / p}\right)$.

To prove Theorem B, we need the following lemmas. The first one is proved by E. Odell, Th. Schlumprecht and A. Zsák.

Lemma 3.5 (Proposition 5.5 in [11]). Let $1<p<\infty$. For a separable reflexive Banach space $X$, the following are equivalent:

(a) $X$ satisfies an asymptotic lower- $\ell_{p}$-tree estimate.

(b) There exists $\gamma \in(0,1)$ such that $X$ satisfies a lower- $T\left(\ell_{p}, \gamma\right)$-tree estimate.

Let $\left(v_{i}\right)$ be a sequence in a Banach space. We say that $\left(v_{i}\right)$ satisfies an asymptotic upper- $\ell_{p}$ estimate if there is a $C>0$ so that for every finite sequence of natural numbers $n_{1}<\cdots<n_{m}$ with $m<n_{1}$ and $\left(a_{i}\right)_{i=1}^{m} \subset \mathbb{R}$,

$$
\left\|\sum_{i=1}^{m} a_{i} v_{n_{i}}\right\| \leq C\left(\sum_{i=1}^{m}\left|a_{i}\right|^{p}\right)^{1 / p} .
$$


Lemma 3.6. Let $1<p<\infty$. Let $\left(u_{i}\right)$ be the canonical basis for $\ell_{p, 1}$ and let $\left(v_{i}\right)$ be a sequence in a Banach space which has an asymptotic upper- $\ell_{p}$ estimate. Then $\left(v_{i}\right)$ is dominated by $\left(u_{i}\right)$.

Proof. By definition, we need to prove that there is a $C_{0}>0$ so that for any $\left(a_{i}\right)_{i=1}^{\infty} \subset \mathbb{R}$,

$$
\left\|\sum_{i=1}^{\infty} a_{i} v_{i}\right\| \leq C_{0}\left\|\sum_{i=1}^{\infty} a_{i} u_{i}\right\| .
$$

By scaling and taking small perturbations, we may assume $0<\left|a_{i}\right| \leq 1$, $\forall i \in \mathbb{N}$. Let $\left(A_{k}\right)$ be the partition of $\mathbb{N}$ defined as

$$
A_{k}=\left\{i \in \mathbb{N}: 1 / 2^{k}<\left|a_{i}\right| \leq 1 / 2^{k-1}\right\} .
$$

Let $I=\left\{k \in \mathbb{N}:\left|A_{k}\right|>2^{-1} \sum_{j<k}\left|A_{j}\right|\right\}$. There are two cases for $I$, finite or infinite. Since the proof when $I$ is finite is essentially the same as when $I$ is infinite, here we just give the proof for the case when $I$ is infinite.

Let $I=\left\{m_{1}, m_{2}, \ldots\right\}$, where $m_{1}<m_{2}<\cdots$. Set $m_{0}=1$ and let $B_{n}=\bigcup_{k=m_{n}}^{m_{n+1}-1} A_{k}$ for $n \geq 0$. Let $C$ be a constant associated with the weak asymptotic upper- $\ell_{p}$ estimate for $\left(v_{i}\right)$. Suppose $2^{t_{n}} \leq\left|A_{m_{n}}\right|<2^{t_{n}+1}$. Then

$$
\begin{aligned}
\left\|\sum_{i \in A_{m_{n}}} a_{i} v_{i}\right\| & \leq \frac{C}{2^{m_{n}-1}}\left(2^{t_{n} / p}+2^{\left(t_{n}-1\right) / p}+\cdots+1\right) \leq \frac{C}{2^{m_{n}-1}} \frac{2^{t_{n} / p}}{1-2^{-1 / p}} \\
& \leq \frac{C}{1-2^{-1 / p}} \frac{1}{2^{m_{n}-1}}\left|A_{m_{n}}\right|^{1 / p} .
\end{aligned}
$$

Noticing that for all $m_{n}<k<m_{n+1}$,

$$
\left|A_{k}\right| \leq(3 / 2)^{k-m_{n}}\left|A_{m_{n}}\right|
$$

we get

$$
\begin{aligned}
\sum_{k=m_{n}+1}^{m_{n+1}-1} \frac{1}{2^{k-1}}\left|A_{k}\right|^{1 / p} & \leq \sum_{k=m_{n}+1}^{m_{n+1}-1} \frac{1}{2^{k-1}}(3 / 2)^{\left(k-m_{n}\right) / p}\left|A_{m_{n}}\right|^{1 / p} \\
& =\sum_{k=m_{n}+1}^{m_{n+1}-1} \frac{1}{2^{m_{n}-1}} \frac{(3 / 2)^{\left(k-m_{n}\right) / p}}{2^{k-m_{n}}}\left|A_{m_{n}}\right|^{1 / p}
\end{aligned}
$$

Now let

$$
\widetilde{C}_{p}=\frac{C}{1-2^{-1 / p}}\left(1+\sum_{k=m_{n}+1}^{\infty} \frac{(3 / 2)^{\left(k-m_{n}\right) / p}}{2^{k-m_{n}}}\right)=\frac{C}{1-2^{-1 / p}}\left(1+\sum_{k=1}^{\infty} \frac{(3 / 2)^{k / p}}{2^{k}}\right) .
$$

We have

$$
\left\|\sum_{i \in B_{n}} a_{i} v_{i}\right\| \leq \sum_{k=m_{n}}^{m_{n+1}-1}\left\|\sum_{i \in A_{k}} a_{i} v_{i}\right\| \leq \frac{1}{2^{m_{n}-1}} \widetilde{C}_{p}\left|A_{m_{n}}\right|^{1 / p}
$$


Next, we are going to estimate $\sum_{k=\sum_{j=1}^{m_{n}-1}\left|A_{j}\right|+1}^{\sum_{j n}^{m_{n}}\left|A_{j}\right|} k^{1 / p-1}$. From the definition of $A_{m_{n}},\left|A_{m_{n}}\right|$ is always greater than or equal to 1 . If $\left|A_{m_{n}}\right|=1$, then $\sum_{j=1}^{m_{n}-1}\left|A_{j}\right|$ can only be 0 or 1 . In either case,

$$
\sum_{k=\sum_{j=1}^{m_{n}-1}\left|A_{j}\right|+1}^{\sum_{j=1}^{m_{n}}\left|A_{j}\right|} k^{1 / p-1} \geq 2^{1 / p-1} \geq\left(3^{1 / p}-(5 / 2)^{1 / p}\right)\left|A_{m_{n}}\right|^{1 / p} .
$$

If $\left|A_{m_{n}}\right| \geq 2$, then

$$
\begin{aligned}
\sum_{k=\sum_{j=1}^{m_{n}-1}\left|A_{j}\right|+1}^{\sum_{j=1}^{m_{n}}\left|A_{j}\right|} k^{1 / p-1} & \geq p\left(\left(\sum_{j=1}^{m_{n}}\left|A_{j}\right|\right)^{1 / p}-\left(\sum_{j=1}^{m_{n}-1}\left|A_{j}\right|+1\right)^{1 / p}\right) \\
& \geq p\left(\left(3\left|A_{m_{n}}\right|\right)^{1 / p}-\left(2\left|A_{m_{n}}\right|+1\right)^{1 / p}\right) \\
& \geq p\left(3^{1 / p}-\left(2+\left|A_{m_{n}}\right|^{-1}\right)^{1 / p}\right)\left|A_{m_{n}}\right|^{1 / p} \\
& \geq p\left(3^{1 / p}-(5 / 2)^{1 / p}\right)\left|A_{m_{n}}\right|^{1 / p} \\
& \geq\left(3^{1 / p}-(5 / 2)^{1 / p}\right)\left|A_{m_{n}}\right|^{1 / p} .
\end{aligned}
$$

We get the first step by using the definite integral of the function $x^{1 / p-1}$. The second step follows from the fact that $(x+a)^{1 / p}-x^{1 / p}$ is a decreasing function for $p>1$, where $a$ is a positive constant. So for any $n \in \mathbb{N}$, we have

$$
\sum_{k=\sum_{j=1}^{m_{n}-1}\left|A_{j}\right|+1}^{\sum_{j=1}^{m_{n}}\left|A_{j}\right|} k^{1 / p-1} \geq\left(3^{1 / p}-(5 / 2)^{1 / p}\right)\left|A_{m_{n}}\right|^{1 / p} .
$$

Hence,

$$
\begin{aligned}
\left\|\sum_{i=1}^{\infty} a_{i} u_{i}\right\| & \geq \sum_{n=1}^{\infty} \frac{1}{2^{m_{n}}}\left(\sum_{k=\sum_{j=1}^{m_{n}-1}\left|A_{j}\right|+1}^{\sum_{j=1}^{m_{n}}\left|A_{j}\right|} k^{1 / p-1}\right) \\
& \geq \sum_{n=1}^{\infty} \frac{1}{2^{m_{n}}}\left(3^{1 / p}-(5 / 2)^{1 / p}\right)\left|A_{m_{n}}\right|^{1 / p} \\
& \geq \frac{3^{1 / p}-(5 / 2)^{1 / p}}{2 \widetilde{C}_{p}} \sum_{n=1}^{\infty}\left\|\sum_{i \in B_{n}} a_{i} v_{i}\right\| \geq \frac{3^{1 / p}-(5 / 2)^{1 / p}}{2 \widetilde{C}_{p}}\left\|\sum_{i=1}^{\infty} a_{i} v_{i}\right\| .
\end{aligned}
$$

The first step above holds because we only compute the contribution of those $a_{i} u_{i}$ 's with $a_{i} \in A_{m_{n}}$. This finishes the proof.

Lemma 3.7. Let $1<p<\infty$ and let $X$ be a separable reflexive Banach space. Let $T$ be a bounded linear operator from $X$ into a Banach space $Y$. Then $(\mathrm{a}) \Rightarrow(\mathrm{b})$, where

(a) $T$ satisfies an asymptotic upper- $\ell_{p}$-tree estimate,

(b) $T$ satisfies an upper- $\ell_{p, 1}$-tree estimate. 
Proof. Since $X$ is a separable reflexive Banach space, by Zippin's theorem [13], we can assume that $X$ is a subspace of a reflexive space with an FDD $\left(E_{n}\right)$. Let $C>0$ be the constant associated with the asymptotic upper- $\ell_{p}$-tree estimate. For $k \in \mathbb{N}$, let

$$
\mathcal{A}^{k}=\left\{\left(x_{i}\right) \in S_{X}^{\omega}:\left\|T\left(\sum_{i=1}^{k} a_{i} x_{i}\right)\right\| \leq C\left(\sum_{i=1}^{k}\left|a_{i}\right|^{p}\right)^{1 / p}, \forall\left(a_{i}\right)_{i=1}^{k} \subset \mathbb{R}\right\} .
$$

Let $\varepsilon>0$ be so small that for all $k \in \mathbb{N}$,

$$
\overline{\mathcal{A}_{\varepsilon}^{k}} \subset\left\{\left(x_{i}\right) \in S_{X}^{\omega}:\left\|T\left(\sum_{i=1}^{k} a_{i} x_{i}\right)\right\| \leq 2 C\left(\sum_{i=1}^{k}\left|a_{i}\right|^{p}\right)^{1 / p}, \forall\left(a_{i}\right)_{i=1}^{k} \subset \mathbb{R}\right\} .
$$

Let $\left(E_{i}^{0}\right)=\left(E_{i}\right)$. Applying Lemma 3.2, we get a decreasing null sequence $\delta^{1}=\left(\delta_{i}^{1}\right)$ and a blocking $\left(E_{i}^{1}\right)$ of $\left(E_{i}^{0}\right)$ so that if $\left(x_{i}\right) \subset S_{X}$ is a $\delta^{1}$-skipped block sequence of $\left(E_{i}^{1}\right)$, then $\left(x_{i}\right)$ lies in $\overline{\mathcal{A}_{\varepsilon}^{1}}$. By repeating this procedure, we obtain decreasing null sequences $\delta^{k}=\left(\delta_{i}^{k}\right)$ and blockings $\left(E_{i}^{k}\right)$ of $\left(E_{i}^{k-1}\right)$ so that if $\left(x_{i}\right) \subset S_{X}$ is a $\left(\delta_{i}^{k}\right)$-skipped block sequence of $\left(E_{i}^{k}\right)$, then $\left(x_{i}\right)$ lies in $\overline{\mathcal{A}_{\varepsilon}^{k}}$. Let $E_{k}^{k}=\bigoplus_{i=n_{k}}^{m_{k}} E_{i}$, and let $\left(F_{i}\right)$ be a blocking of $\left(E_{i}\right)$ so that $F_{k}=\bigoplus_{i=n_{k}}^{n_{k+1}-1} E_{i}$. We can then choose a decreasing null sequence $\delta=\left(\delta_{i}\right)$ so that if $\left(x_{i}\right) \subset S_{X}$ is a $\delta$-skipped block sequence of $\left(F_{i}\right)$, then $\left(x_{i}\right)$ is a basic sequence and any normalized block sequence $\left(z_{i}\right)_{i=1}^{k}$ of $\left(x_{i}\right)_{i=k}^{\infty}$ is a $\delta^{k}$-skipped block sequence of $\left(E_{i}^{k}\right)$. Hence, by Lemma 3.6, $\left(T x_{i}\right)$ is dominated by the canonical basis of $\ell_{p, 1}$. This shows $T$ satisfies an upper- $\ell_{p, 1}$-tree estimate.

Proof of Theorem B. By Lemma 3.5, $X$ satisfies a lower- $T\left(\ell_{q}, \gamma\right)$-tree estimate for some $0<\gamma<1$. By Lemma 3.7, $T$ satisfies an upper- $\ell_{p 1}$-tree estimate. Since the canonical basis of $T\left(\ell_{q}, \gamma\right)$ dominates the canonical basis of $\ell_{r}$ and the canonical basis of $\ell_{r}$ dominates the canonical basis of $\ell_{p 1}$ when $1<q<r<p<\infty$, by Theorem 3.1, we deduce that $T$ factors through a subspace of $\left(F_{n}\right)_{\ell_{r}}$, where $F=\left(F_{n}\right)$ is a sequence of finite-dimensional spaces.

By Theorem B, we have

COROLlary 3.8. Let $1<q<p<\infty$ and let $X$ be a reflexive asymptotic $\ell_{q}$ space. Let $T$ be a bounded linear operator from $X$ which satisfies an asymptotic upper- $\ell_{p}$-tree estimate. Then $T$ factors through a subspace of a space with an $\left(\ell_{p}, \ell_{q}\right) F D D$.

REMARK 3.9. Theorem B and Corollary 3.8 start with asymptotic conditions while end up with factorizations through subspaces of spaces with properties much stronger than asymptotic properties. This gives us some information on the relations between asymptotic $\ell_{p}$ spaces and $\left(F_{n}\right)_{\ell_{r}}$ spaces. However, they do not tell us what happens when $p=q$. 
The following theorem provides a result for the special case when $p=q$.

TheOREm 3.10. Let $2<p<\infty$. Let $X$ be a separable reflexive asymptotic $\ell_{p}$ space. Let $T$ be a bounded linear operator from $X$ into $L_{p}$ which satisfies an asymptotic upper- $\ell_{p}$-tree estimate. Then $T$ factors through $\ell_{p}$.

Proof. W. B. Johnson proved in [2] that for $p>2$, a bounded linear operator $T$ into $L_{p}$ factors through $\ell_{p}$ if and only if $T$ is compact when considered as an operator into $L_{2}$. So it is enough to show that $T$ is compact as an operator into $L_{2}$. By Corollary 4.8 in [11], $X$ embeds into a reflexive Banach space with an asymptotic $\ell_{p} \operatorname{FDD}\left(E_{n}\right)$. Let $\left(h_{n}\right)$ be the canonical Haar basis of $L_{2}$. If $T$ is not compact as an operator into $L_{2}$, then there are a $\delta>0$ and a perturbation of a normalized block sequence $\left(x_{i}\right)$ with respect to $\left(E_{n}\right)$ so that $\left(i_{p, 2} \circ T x_{i}\right)$ is essentially a block sequence with respect to $\left(h_{n}\right)$ and $\left\|i_{p, 2} \circ T x_{i}\right\|>\delta, \forall i \in \mathbb{N}$, where $i_{p, 2}$ is the formal identity map from $L_{p}$ into $L_{2}$. This gives a contradiction since, on the normalized weakly null tree $\left(x_{A}\right)_{A \in[\mathbb{N}]<\omega}, x_{A}=x_{\max \{A\}}, T$ does not satisfy an asymptotic upper- $\ell_{p}$-tree estimate.

REMARK 3.11. Theorem 3.10 holds even if we only assume that for every normalized weakly null sequence in $X$ there is a subsequence whose image under $T$ satisfies an asymptotic upper- $\ell_{r}$ estimate for some $2<r<\infty$.

Acknowledgments. The author thanks E. Odell for useful conversations and the referee for careful reading.

\section{References}

[1] P. G. Casazza and T. J. Shura, Tsirelson's Space, Lecture Notes in Math. 1363, Springer, Berlin, 1989.

[2] W. B. Johnson, Operators into $L_{p}$ which factor through $l_{p}$, J. London Math. Soc. (2) 14 (1976), 333-339.

[3] -, On quotients of $L_{p}$ which are quotients of $\ell_{p}$, Compos. Math. 34 (1977), 69-89.

[4] W. B. Johnson and M. Zippin, On subspaces of quotients of $\left(\sum G_{n}\right)_{\ell_{p}}$ and $\left(\sum G_{n}\right)_{c_{0}}$, Israel J. Math. 13 (1972), 311-316.

[5] - - - Subspaces and quotient spaces of $\left(\sum G_{n}\right)_{l_{p}}$ and $\left(\sum G_{n}\right)_{c_{0}}$, ibid. 17 (1974), $50-55$.

[6] J. Lindenstrauss and L. Tzafriri, Classical Banach Spaces I. Sequence Spaces, Springer, Berlin, 1977.

[7] B. Maurey, V. D. Milman and N. Tomczak-Jaegermann, Asymptotic infinite-dimensional theory of Banach spaces, in: Oper. Theory Adv. Appl. 77, Birkhäuser, Basel, 1995, 149-175.

[8] V. D. Milman and N. Tomczak-Jaegermann, Asymptotic $\ell_{p}$ spaces and bounded distortions, in: Contemp. Math. 144, Amer. Math. Soc., Providence, RI, 1993, 173195.

[9] E. Odell and Th. Schlumprecht, Trees and branches in Banach spaces, Trans. Amer. Math. Soc. 354 (2002), 4085-4108. 
[10] E. Odell and Th. Schlumprecht, A universal reflexive space for the class of uniformly convex Banach spaces, Math. Ann. 335 (2006), 901-916.

[11] E. Odell, Th. Schlumprecht and A. Zsák, On the structure of asymptotic $\ell_{p}$ spaces, Quart. J. Math., to appear.

[12] B. Zheng, On operators which factor through $\ell_{p}$ or $c_{0}$, Studia Math. 176 (2006), 177-190.

[13] M. Zippin, Banach spaces with separable duals, Trans. Amer. Math. Soc. 310 (1988), $371-379$.

Department of Mathematics

The University of Texas at Austin

1 University Station C1200

Austin, TX 78712-0257, U.S.A.

E-mail: btzheng@math.utexas.edu

Received August 13, 2007

Revised version December 24, 2007 